\title{
Robust CPHD Fusion for Distributed Multitarget Tracking Using Asynchronous Sensors
}

\author{
Benru Yu, Tiancheng Li, Member, IEEE, and Hong Gu
}

\begin{abstract}
This paper concentrates on tracking multiple targets using an asynchronous network of sensors with different sampling rates. First, a timely fusion approach is proposed for handling measurements from asynchronous sensors. In the proposed approach, the arithmetic average fusion of the estimates provided by local cardinalized probability hypothesis density filters is recursively carried out according to the network-wide sampling time sequence. The corresponding intersensor communication is conducted by a partial flooding protocol, in which either cardinality distributions or intensity functions pertinent to local posteriors are disseminated among sensors. Moreover, both feedback and non-feedback fusion-filtering modes are provided to meet the performance and real-time requirements, respectively. Second, an extension of the timely fusion approach referred to as robust bootstrap approach is presented, which can deal with unknown clutter and detection parameters by exploiting a local bootstrap filtering scheme. Finally, numerical simulations are performed to test the proposed approaches.
\end{abstract}

Index Terms-Multitarget tracking, arithmetic average fusion, asynchronous sensors, flooding protocol, different sampling rates, bootstrap filtering, random finite sets.

\section{INTRODUCTION}

$\mathbf{N}$ OWADAYS, sensor networks are widespread in military and civilian fields [1], [2] thanks to the advances in wireless communication and sensors techniques. In particular, the distributed sensor network has attracted substantial attention owing to the advantages of high reliability, strong robustness and excellent scalability over its centralized counterpart. A significant topic in the distributed sensor network is distributed multitarget tracking (DMT) [3]-[6], which involves tracking stochastic appearing and disappearing targets using decentralized but interconnected sensors in a collaborative manner. The main concerns of DMT are twofold: 1) how to obtain local estimates at each sensor from cluttered and noisy sensors observations; 2) how to fuse estimates provided by different sensors in the presence of unknown and ubiquitous intersensor cross-correlation.

The random finite set (RFS) approach [7], [8] provides a rigorous and promising framework for the first concern of which the centerpiece lies in the multitarget Bayes (MTB) filter. Unfortunately, the MTB filter is computationally intractable in general, and approximate solutions are therefore needed. The probability density hypothesis (PHD) [9] and cardinalized

Benru Yu and Hong $\mathrm{Gu}$ are with the School of Electronic and Optical Engineering, Nanjing University Of Science and Technology, Nanjing 210094 China (e-mail: nusteoeybr@163.com; ghstudent_rceet@126.com).

Tiancheng Li is with the Key Laboratory of Information Fusion Technology (Ministry of Education), School of Automation, Northwestern Polytechnical University, Xi'an 710072, China (e-mail: t.c.li@nwpu.edu.cn)
PHD (CPHD) [10] filters are tractable approximations of the MTB filter. Unlike the PHD filter, which merely propagates the intensity function, the CPHD filter additionally propagates the cardinality distribution, which consequently achieve preferable performance. Practical implementations of the two filters have been reported in [11]-[13]. These results render the PHD and CPHD filters accessible to a wider audience.

Aiming at the second concern, two average fusion methods, i.e., arithmetic average (AA) [14]-[16] and geometric average (GA) [3], [4], [17], [18], have been proposed, which are immune to the correlations among sensors. Compared with the GA fusion, the AA fusion has much lower computational complexity [19]. This merit makes the AA fusion suitable for realistic sensor networks with limited computing resources. Furthermore, the AA fusion of RFS densities leads to a mixture distribution and it admits the closed-form MarkovBayesian recursion [20]. With these facts in mind, we focus on the AA fusion in this work. Recently, the AA fusion has been applied in conjunction with the PHD and CPHD filters in various scenarios, demonstrating outstanding performance not only in the context of target tracking [16], [21]-[27] but also vehicular network positioning [28] and multi-robot collaboration [29].

Most of the existing distributed fusion approaches depend on the ideal assumption that the sampling rates of sensors are identical. In fact, even in the simplest homogeneous network, communication delay is unavoidable, which poses the need for asynchronous fusion. Several multisensor PHD filters [30]-[32] have been proposed for asynchronous radars with different sampling rates. Unfortunately, these filters merely suit scenarios with few sensors. Extensions to an arbitrary number of sensors have been studied in [33], [34], where the fusion of local estimates are performed in accordance with a fixed period via the central node. A robust PHD fusion algorithm that is capable of joint estimating target states and time-offsets among sensors has been investigated in [35]. However, estimating time-offsets at sensors using a sampling-based optimization strategy requires extra consumption of the communication and computing resources and means limited applicability to the energy-constrained sensor network. The works mentioned above are all on the foundation of the PHD filter, which can be treated as a special case of the CPHD filter. Furthermore, none of these works takes unknown clutter and detection parameters into account.

When unknown model parameters are involved, robust PHD and CPHD filters have been presented in [36]-[38], which are able to estimate the clutter and detection parameters while filtering. Further improvements have been reported in [39]- 
[41]. Adaptive PHD and CPHD filters relying on the inverse gamma Gaussian mixture (GM) model have been given in [42], where the non-Gaussian feature parameters are utilized in addition to the position information in order to adapt the timevarying detection probability. Robust estimators [43]-[45] under the PHD filtering framework have also been presented to simultaneously estimate the multitarget state and clutter rate. However, these filters are designed for the single sensor case.

Based on the analyses mentioned above, we present the first ever distributed CPHD filter that is able to deal with different sampling rates among sensors and unknown clutter and detection parameters in this paper. The main contributions are summarized below.

1) We propose a timely fusion approach to DMT based on asynchronous sensors, where potential communication delay among sensors is allowable. In the proposed approach, the AA fusion of local CPHD estimates is performed as long as new measurements have been received by any sensor.

2) We adopt a partial flooding protocol for intersensor communication, in which merely local posteriors are disseminated among sensors. At each flooding iteration, the information exchanged between neighbors can either be the individual cardinality distribution or the pairwise cardinality distribution and intensity function.

3) We provide both feedback and non-feedback fusionfiltering modes with the intention of achieving preferable performance and reducing/avoiding sensor data delay, respectively. In the feedback mode, the local estimates are improved through fusion, whereas the fusion outcome does nothing help to the local estimates in the nonfeedback mode.

4) We extend the timely fusion approach to handle unknown model parameters by exploiting a local bootstrap filter consisting of a PHD estimator and a CPHD filter. In particular, the PHD estimator provides the clutter and detection parameters for the CPHD filter, while the CPHD filter generates local estimates.

The paper is outlined as follows. Section II gives the relevant preliminaries. Sections III and IV detail the proposed timely fusion and robust bootstrap approaches, respectively. Section V presents numerical studies. Section VI concludes this paper.

\section{Preliminaries}

\section{A. Background on RFS}

An RFS is essentially a set-valued random variable of which the randomness is captured by its probability density. Let $\mathcal{X}$ be an RFS and $f(\mathcal{X})$ be its probability density. The cardinality distribution of $\mathcal{X}$ denoted by $\rho(n)$ stands for the probability of the event that $\mathcal{X}$ comprises $n$ elements. Another crucial statistic of $\mathcal{X}$ is the intensity function $\nu(x)$, whose integral, i.e., $\int_{x \in \mathcal{X}} \nu(x) d x$, stands for the expected number of elements in $\mathcal{X}$. Definitions of the cardinality distribution and intensity function have been explicitly given in [7], [8].
We specify two kinds of RFSs used throughout this paper. An identical independently distributed cluster (IIDC) RFS $\mathcal{Y}$ has the probability density

$$
f(\mathcal{Y})=n ! \rho(n) \prod_{y \in \mathcal{Y}} s(y) .
$$

where $s(y)$ represents the spatial distribution. The corresponding intensity function is given by

$$
\nu(y)=\sum_{n=0}^{\infty} n \rho(n) s(y) .
$$

An IIDC RFS reduces to a Poisson RFS when the cardinality is Poisson distributed. Hence, the probability density and intensity function of a Poisson RFS $\mathcal{Z}$ can be respectively expressed as

$$
\begin{aligned}
f(\mathcal{Z}) & =e^{-\lambda} \prod_{z \in \mathcal{Z}} \lambda s(z), \\
\nu(z) & =\lambda s(z),
\end{aligned}
$$

where $\lambda$ denotes the mean of the Poisson distribution.

The Poisson and IIDC RFSs are closely related to the PHD and CPHD filters. To be specific, the intensity function of a Poisson RFS describing the multitarget state is recursively propagated in the PHD filter [9]. Whereas the CPHD filter [10] models the multitarget state as an IIDC RFS and jointly propagates its cardinality distribution and intensity function as time progresses.

\section{B. Multisensor Multitarget System Model}

Assume that there are $N_{k}$ targets with states $x_{k}^{i}, i=$ $1, \cdots, N_{k}$ in the scene at time $k$. The current multitarget state can then be modeled by an RFS $\mathcal{X}_{k}=\left\{x_{k}^{1}, \cdots, x_{k}^{N_{k}}\right\}$. In this paper, $X_{k}$ is formed by

$$
\mathcal{X}_{k}=\left[\bigcup_{x_{k-1} \in \mathcal{X}_{k-1}} \mathcal{S}_{k}\left(x_{k-1}\right)\right] \cup \mathcal{B}_{k},
$$

where

$$
\mathcal{S}_{k}\left(x_{k-1}\right)= \begin{cases}\left\{x_{k}\right\}, & \text { if } x_{k-1} \text { continues to exist } \\ \emptyset, & \text { if } x_{k-1} \text { vanishes }\end{cases}
$$

and $\mathcal{B}_{k}$ is a Poisson RFS modeling newborn targets. It is supposed that newborn targets and existing targets are mutually independent and that the transition of single target state from $x_{k-1}$ to $x_{k}$ is determined by the Markov density $\varphi_{k \mid k-1}\left(x_{k} \mid x_{k-1}\right)$.

A sensor network consisting of $S$ sensors with different sampling rates $T_{1}, \cdots, T_{S}$ is considered. Each sensor is capable of acquiring measurement data, processing local information and communicating with neighbors. Mathematically, the sensor network can be represented by $(\mathcal{E}, \mathcal{L})$, where $\mathcal{E}=\{1, \cdots, S\}$ and $\mathcal{L} \subseteq \mathcal{E} \times \mathcal{E}$ denote the set of sensors and the set of communication links, respectively. The diameter of the graph is denoted by $D_{m}$. The sampling time instants (STIs) of all sensors are available and collected by $\mathcal{T}=\bigcup_{i=1}^{S} \mathcal{T}_{i}$, where $\mathcal{T}_{i}=\left\{\tau_{i}+m T_{i}, m \in \mathbb{N}^{+}\right\}$and $\tau_{i}$ denotes the initial 
STI of sensor $i$. If sensor $i$ can acquire measurement data at time $k$, i.e., $k \in \mathcal{T}_{i}$, the multitarget measurement at sensor $i$ and time $k$ can then be formulated by

$$
\mathcal{Z}_{i, k}=\left[\bigcup_{x_{k} \in \mathcal{X}_{k}} \mathcal{D}_{i, k}\left(x_{k}\right)\right] \cup \mathcal{C}_{i, k}
$$

where

$$
\mathcal{D}_{i, k}\left(x_{k}\right)= \begin{cases}\left\{z_{i, k}\right\}, & \text { if } x_{k} \text { detected } \\ \emptyset, & \text { if } x_{k} \text { misdetected }\end{cases}
$$

and the detection probability is denoted by $p_{i, k}^{d}\left(x_{k}\right)$. If detected, a target-originated measurement $z_{i, k}$ is generated in accordance with the likelihood function $\psi_{i, k}\left(z_{i, k} \mid x_{k}\right) . \mathcal{C}_{i, k}$ is a Poisson RFS modeling clutter measurements (not originating from any target). The intensity function of $\mathcal{C}_{i, k}$ is given by $\kappa_{i, k}=\lambda_{i, c} / V_{i}$, where $\lambda_{i, c}$ and $V_{i}$ denote the clutter rate and volume of the field of view (FOV), respectively. The clutter measurements and target-originated measurements are not related to each other.

\section{AA Fusion}

The AA fusion of $S$ multitarget probability distributions ${ }^{1}$ (MPDs) $f_{1}(\mathcal{X}), \cdots, f_{S}(\mathcal{X})$ is given by

$$
\bar{f}(\mathcal{X})=\sum_{i=1}^{S} w_{i} f_{i}(\mathcal{X}),
$$

where $w_{i}$ denotes the positive fusing weight associated with sensor $i$ and $\sum_{i=1}^{S} w_{i}=1$.

As indicated in [46], [47], the AA fusion can be interpreted in the sense of Kullback-Leibler divergence (KLD). That is,

$$
\bar{f}(\mathcal{X})=\underset{g}{\arg \min } \sum_{i=1}^{S} w_{i} D_{\mathrm{KL}}\left(f_{i} \| g\right),
$$

where $D_{\mathrm{KL}}(f \| g)$ stands for the KLD between MPDs $f(\mathcal{X})$ and $g(\mathcal{X})$, which can be computed as

$$
D_{\mathrm{KL}}(f \| g)=\int f(\mathcal{X}) \log \frac{f(\mathcal{X})}{g(\mathcal{X})} \delta \mathcal{X} .
$$

It has also been proved in [47], [48] that the AA fusion is a Fréchet mean as follows:

$$
\bar{f}(\mathcal{X})=\underset{g}{\arg \min } \sum_{i=1}^{S} w_{i}\left\|f_{i}-g\right\|^{2},
$$

where $\|f\|^{2} \triangleq \int(f(\mathcal{X}))^{2} \delta \mathcal{X}$.

Recently, Li [20] pointed out that for MPDs $f_{i}(\mathcal{X}), i=$ $1, \cdots, S$, the KLD of any MPD $g(\mathcal{X})$ relative to their AA fusion $\bar{f}(\mathcal{X})$ satisfies

$$
\begin{aligned}
D_{\mathrm{KL}}(\bar{f} \| g) & =\sum_{i=1}^{S} w_{i}\left(D_{\mathrm{KL}}\left(f_{i} \| g\right)-D_{\mathrm{KL}}\left(f_{i} \| \bar{f}\right)\right), \\
& \leq \sum_{i=1}^{S} w_{i} D_{\mathrm{KL}}\left(f_{i} \| g\right),
\end{aligned}
$$

\footnotetext{
${ }^{1}$ When an RFS is employed to model the multitarget state, its probability density is known as multitarget probability distribution.
}

where the equation holds if and only if $f_{1}(\mathcal{X}), \cdots, f_{S}(\mathcal{X})$ are identical. Let $g(\mathcal{X})$ be the target distribution, then we can observe from (13) that the AA fusion of a number of MPDs matches the target distribution better than all MPDs on average. This offers an information-theoretic justification for the AA fusion.

The following Lemma presents theoretical results of the AA fusion of IIDC MPDs.

Lemma 1: Given fusing IIDC MPDs $f_{i}(\mathcal{X}), i=1, \cdots, S$ and positive fusing weights $w_{1}, \cdots, w_{S}$ subject to $\sum_{i=1}^{S} w_{i}=$ 1. Their AA fusion $\bar{f}(\mathcal{X})$ has the cardinality distribution $\bar{\rho}(n)=\sum_{i=1}^{S} w_{i} \rho_{i}(n)$ and intensity function $\bar{\nu}(x)=$ $\sum_{i=1}^{S} w_{i} \nu_{i}(x)$. Unfortunately, $\bar{f}(\mathcal{X})$ is not an IIDC MPD. To maintain the closure property, an IIDC MPD approximation of $\bar{f}(\mathcal{X})$ is given by

$$
\tilde{f}(\mathcal{X})=n ! \bar{\rho}(n) \prod_{x=1}^{S} \bar{s}(x),
$$

where $\bar{s}(x)=\bar{\nu}(x) / \sum_{n=0}^{\infty} n \bar{\rho}(n)$ and the intensity function as well as the cardinality distribution of $\tilde{f}(\mathcal{X})$ and $\bar{f}(\mathcal{X})$ are equivalent. Mathematical proofs of Lemma 1 have been given in [16], [46] and are therefore omitted here.

\section{Proposed Timely Fusion ApproACH}

In the timely fusion approach, the AA fusion of local CPHD estimates is carried out at each sensor $i \in \mathcal{E}$ and fusion time instant (FTI) $k \in \mathcal{T}$. A simple example is given in Fig. 1 to illustrate the timely fusion mechanism. It can be clearly seen that sensor S5 performs fusion at not only its own but also other sensors' STIs. In fact, the FTIs of sensors S1-S4 are consistent with those of sensor S5. The timely fusion approach comprises filtering, communication and fusion three steps. In what follows, we detail these steps in order.

\section{A. Local Multitarget Filtering}

Due to different sampling rates among sensors, measurements from distinct sensors are often time-misaligned. Hence, merely part of the sensors can receive measurement data at a generic FTI $k$. We name these sensors as measurement available sensors (MASs) and the remaining sensors as measurement unavailable sensors (MUSs). Correspondingly, the sensor set $\mathcal{E}$ can be decomposed into two subsets $\mathcal{E}_{a, k}$ and $\mathcal{E}_{u, k}$ with constraints $\mathcal{E}=\mathcal{E}_{a, k} \cup \mathcal{E}_{u, k}$ and $\mathcal{E}_{a, k} \cap \mathcal{E}_{u, k}=\emptyset$. Here, $\mathcal{E}_{a, k}$ and $\mathcal{E}_{u, k}$ stand for the set of MASs and the set of MUSs, respectively. At time $k$, each sensor $i$ carries out a single sensor CPHD filter to get its local cardinality distribution $\rho_{i, k}(n)$ and intensity function $\nu_{i, k}(x)$. If $i \in \mathcal{E}_{a, k}, \rho_{i, k}(n)$ and $\nu_{i, k}(x)$ are obtained by sequential performing the single sensor CPHD prediction and update stages, otherwise, $\nu_{i, k}(x)$ and $\rho_{i, k}(n)$ are obtained by performing the single sensor CPHD prediction stage only. For details on the single sensor CPHD filter, readers are referred to [10], [12].

We employ the GM method [11], [12] to implement the local CPHD filter. As a result, the intensity function $\nu_{i, k}(x)$ 




sensor topology

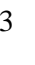



$:$ :TTlof 54

: STI of S2

- : STI of S3

$\bullet$ : STI of S5 •: FTI of S5

Fig. 1. Illustration of the timely fusion mechanism.

is represented by $J_{i, k}$ Gaussian terms (GTs) with positive weights $\alpha_{i, k}^{j}, j=1, \cdots, J_{i, k}$, i.e.,

$$
\nu_{i, k}(x)=\sum_{j=1}^{J_{i, k}} \alpha_{i, k}^{j} \mathcal{N}\left(x ; \mu_{i, k}^{j}, \Sigma_{i, k}^{j}\right),
$$

where $\mathcal{N}(x ; \mu, \Sigma)$ denotes the Gaussian probability density with mean $\mu$ and covariance $\Sigma$. Equivalently, $\nu_{i, k}(x)$ is characterized by the parameter set $\mathcal{G}_{i, k} \triangleq\left\{\left(\alpha_{i, k}^{j}, \mu_{i, k}^{j}, \Sigma_{i, k}^{j}\right)\right\}_{j=1, \cdots, J_{i, k}}$. In principle, the cardinality distribution $\rho_{i, k}(n)$ is computationally infeasible since it involves an infinite number of terms. Fortunately, it is sufficient to truncate $\rho_{i, k}(n)$ at $n=N_{\max }$ and approximate it with $\left\{\rho_{i, k}(n)\right\}_{n=0}^{N_{\max }}[12]$. The estimated target number $\hat{N}_{i, k}$ can be calculated by the mode $\hat{N}_{i, k}=\underset{n}{\arg \max } \rho_{i, k}(n)$ or the mean $\hat{N}_{i, k}=\sum_{j=1}^{J_{i, k}} \alpha_{i, k}^{j}$. Since the mode is superior to the mean in general, it can be used to modify the weights of GTs as done in (19).

Abiding by [27], merely target-like GTs (T-GTs) are disseminated while the remaining false-alarm-like GTs are discarded. This has been proved to be able to not only reduce the computational burden and communication cost but also reduce the adverse effect of the AA fusion. We adopt a threshold rule to identify the T-GTs, where the GTs with weights greater than a preset threshold $r_{\text {gate }}$ are regarded as the T-GTs.

\section{B. Partial Flooding Communication}

We begin with a brief review of the distributed flooding protocol [49], in which each sensor plays a role analogous to a fusion center and collects information from all other sensors through iterative peer to peer (P2P) communication. Under the premise of strongly connected sensor network, complete consensus, namely the information set of each sensor is identical, can be achieved through communication iterations. Determination of the total number of communication iterations has been explicitly investigated in [19]. In spite of its appealing results in theory, the distributed flooding protocol poses severe challenges to the communication and storage capabilities in practice.

In this work, we adopt an efficient flooding protocol for intersenor communication, where only local posteriors are disseminated among sensors. The resulting partial flooding is conditioned on the relevant information from a part of the sensors (more precisely stated, MASs), which differs from the existing flooding protocol conditioned on the information from all sensors. The motivation for the partial flooding is the fact that the local posterior is more accurate than the local prediction from the Bayesian inference perspective.

To gain insights of the consensus of the partial flooding, we encapsulate the information that needs to be transmitted into a set and define the following dynamics:

$$
\mathcal{I}_{i}(\ell+1)=\mathcal{I}_{i}(\ell) \cup \mathcal{U}_{i}(\ell),
$$

where $\mathcal{I}_{i}(\ell)$ and $\mathcal{U}_{i}(\ell)$ stand for the existing and newly received information sets of sensor $i$ at iteration $\ell$, respectively, $\mathcal{I}_{i}(0)$ stands for the initial information set of sensor $i$ satisfying $\forall i \in \mathcal{E},\left|\mathcal{I}_{i}(0)\right|=1$ and $\mathcal{U}_{i}(0)=\emptyset$.

As a result of partial flooding, at any time $k \in \mathcal{F}$, we have the following results:

$$
\begin{aligned}
& \text { 1) } \forall i \in \mathcal{E}_{a, k}, \ell \geq D_{m}, \mathcal{I}_{i}(\ell)=\bigcup_{j \in \mathcal{E}_{a, k}} \mathcal{I}_{j}(0) . \\
& \text { 2) } \forall i \in \mathcal{E}_{u, k}, \ell \geq D_{m}, \mathcal{I}_{i}(\ell)=\bigcup_{j \in \mathcal{E}_{a, k} \cup\{i\}} \mathcal{I}_{j}(0) .
\end{aligned}
$$

Clearly, complete consensus is achieved in MASs and MUSs, respectively.

\section{AA-Based CPHD Fusion}

After the final flooding iteration $L$, the collection of cardinality distributions and intensity functions at sensor $i$ and time $k$ is given by $\left\{\rho_{r, k}(n), \nu_{r, k}(x)\right\}_{r \in \mathcal{M}_{i}(L) \cup\{i\}}$, where $\mathcal{M}_{i}(L)$ stands for the collection of sensors that are no more than $L$ hops away from sensor $i$. Note that $i \notin \mathcal{M}_{i}(L)$. Under the premise of uniform fusion weights, Lemma 1 is realized in the following Proposition.

Proposition 1: The mean cardinality distribution and mean intensity function are respectively computed as follows:

$$
\begin{aligned}
& \bar{\rho}_{i, k}^{[L]}(n)=\frac{1}{\left|\mathcal{M}_{i, k}(L)\right|} \sum_{r \in \mathcal{M}_{i, k}(L)} \rho_{r, k}(n), \\
& \bar{\nu}_{i, k}^{[L]}(x)=\frac{1}{\left|\mathcal{M}_{i, k}(L)\right|} \sum_{r \in \mathcal{M}_{i, k}(L)} \nu_{r, k}(x),
\end{aligned}
$$

where $\mathcal{M}_{i, k}(L)=\mathcal{M}_{i}(L) \cup\{i\} \cap \mathcal{E}_{a, k}$ and the initial conditions are specified by $\bar{\rho}_{i, k}^{[0]}(n)=\rho_{i, k}(n)$ and $\bar{\nu}_{i, k}^{[0]}(n)=\nu_{i, k}(n)$, 
respectively. After pruning and merging steps [11], [12], $\bar{\nu}_{i, k}^{[L]}(x)$ can be represented by the fused GM parameters $\overline{\mathcal{G}}_{i, k} \triangleq\left\{\left(\bar{\alpha}_{i, k}^{j}, \bar{\mu}_{i, k}^{j}, \bar{\Sigma}_{i, k}^{j}\right)\right\}_{j=1, \cdots, \bar{J}_{i, k}}$. The target number estimate $\hat{N}_{i, k}=\underset{n}{\arg \max } \bar{\rho}_{i, k}^{[L]}(n)$ is then exploited to modify the weights $\bar{\alpha}_{i, k}^{j}, j=1, \cdots, \bar{J}_{i, k}$ as follows:

$$
\bar{\alpha}_{i, k}^{j}=\beta_{i} \bar{\alpha}_{i, k}^{j},
$$

where $\beta_{i}=\hat{N}_{i, k} / \sum_{j=1}^{\bar{J}_{i, k}} \bar{\alpha}_{i, k}^{j}$.

The procedures of the AA-CPHD fusion are summarized in Algorithms 1.

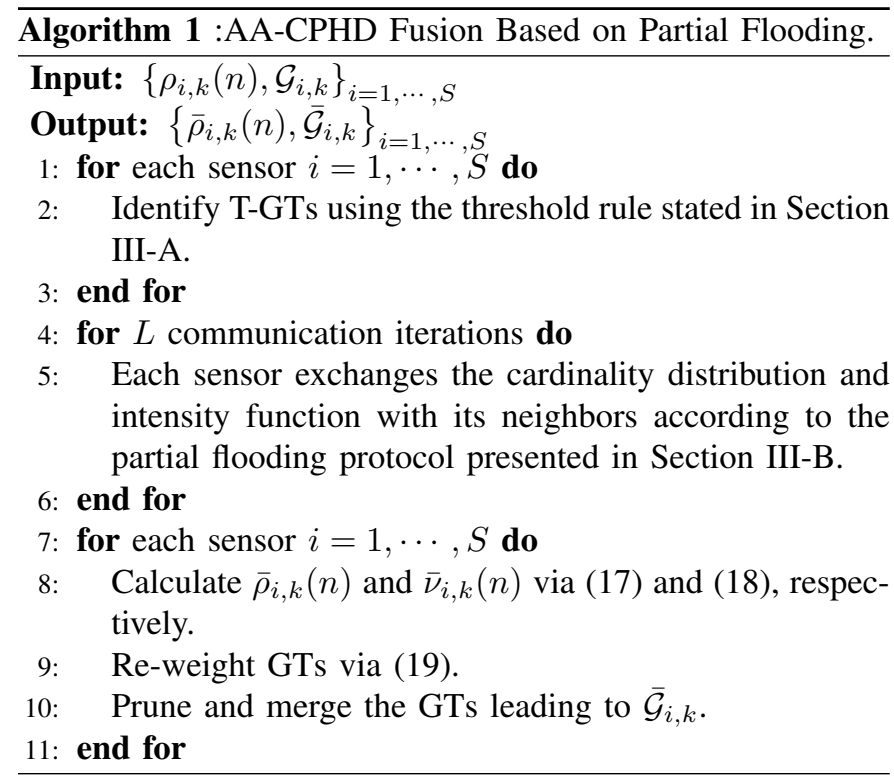

Remark 1: As indicated in Lemma 1, the fusion of $\rho_{i, k}(n)$ is irrelative to $\nu_{i, k}(x)$. It is therefore feasible to disseminate and fuse $\rho_{i, k}(n)$ only. That means merely the cardinality distribution average in (17) is required. Afterward, the target number estimate $\hat{N}_{i, k}$ is exploited to scale the weights of unfused TGCs in a way similar to (19). The resulting approach is named as AA-based cardinality consensus (hereafter abbreviated as AA-CC).

Remark 2: We provide feedback and non-feedback fusionfiltering modes to the AA-CPHD fusion and the AA-CC fusion. In the feedback mode, the fusion outcome takes part in the update of local estimates. When the communication and fusion operations are time-costly due to plenty of reasons such as high sampling rate and large network size, the feedback mode suffers from sensor data delay since the measurement data is unused until the end of the fusion operation. To mitigate this drawback, we adopt a non-feedback alternative [50], in which the fusion outcome does nothing help to the local estimates. A schematic diagram of the two schemes with regard to sensor $i$ and its three successive FTIs $r, s, t$, is presented in Fig. 2, where $r<s<t, r=m T_{i}+\tau_{i}$, and $t=(m+1) T_{i}+\tau_{i}$. As shown, it is the feedback mode rather than the non-feedback mode that improves the local estimates via fusion.

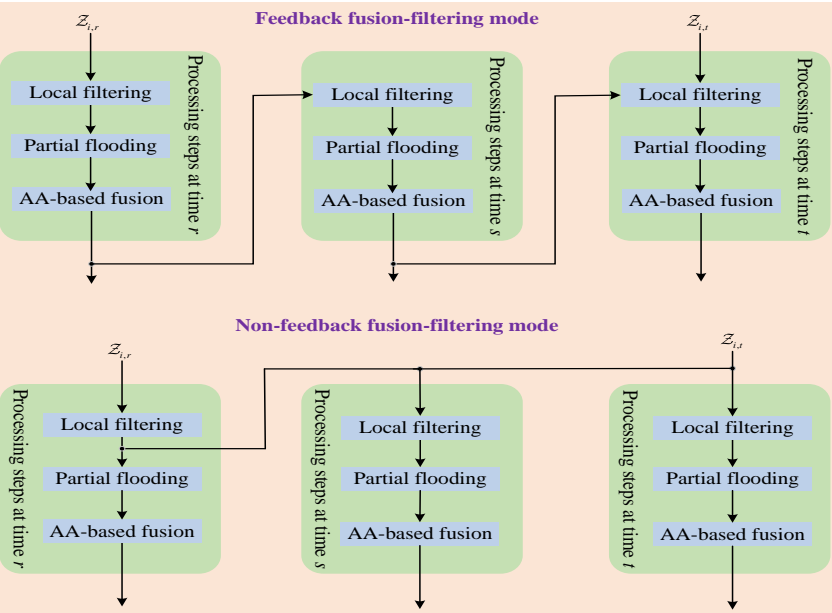

Fig. 2. Schematic diagram of the feedback and non-feedback modes.

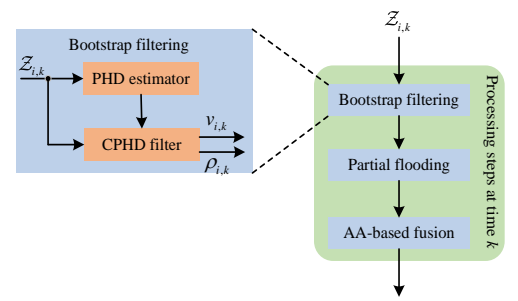

Fig. 3. Schematic diagram of the local bootstrap filtering scheme at sensor $i$ and time $k$.

\section{Extension to UnKnown Model Parameters}

In this section, we focus on the proposed robust bootstrap approach, which extends the timely fusion approach discussed in the previous section to accommodate unknown clutter and detection parameters using the bootstrap filtering scheme analogous to [39], [40]. The basic idea of the scheme is to recursively estimate, at each sensor $i \in \mathcal{E}$ and time $k \in \mathcal{T}_{i}$, the required model parameters by exploiting a local PHD estimator first and then feed its estimates to a local CPHD filter, as demonstrated in Fig. 3. Such a scheme inherits the benefit of the PHD filter regarding linear complexity and the benefit of the CPHD filter in that it provides high quality estimates. What distinguishes the timely fusion approach from the robust bootstrap approach is the local filtering step. Specifically, the former adopts the local CPHD filtering scheme, whereas the bootstrap filtering scheme is employed in the latter. In the following, we present the local bootstrap filtering step for the cases of unknown parameters in clutter and detection, respectively.

\section{A. Unknown Clutter Rate Case}

The centerpiece of dealing with unknown clutter rate is to model clutter as a set of false targets (distinct from actual targets). Both false and actual targets follow standard dynamic and measurement models. The multitarget state is therefore formulated by a hybrid RFS, which consists of a disjoint union of the RFSs associated with actual and false targets. Estimating the hybrid RFS yields the number and state information on actual targets together with the clutter rate. 
The PHD filter with unknown clutter rate (hereafter referred to as $\lambda$-PHD filter) recursively propagates the intensity function $\breve{\nu}_{k}(\breve{x})$ of the hybrid RFS $\mathcal{X}_{k}=\mathcal{X}_{k}^{f} \uplus \mathcal{X}_{k}^{a}$, which can be decomposed into

$$
\breve{\nu}_{k}(\breve{x})=\left\{\begin{array}{ll}
\nu_{k}^{f}(c), & \breve{x}=c \\
\nu_{k}^{a}(x), & \breve{x}=x
\end{array},\right.
$$

where $\nu_{k}^{f}(\cdot)$ and $\nu_{k}^{a}(\cdot)$ denote the intensity functions for the false target RFS $\mathcal{X}_{k}^{f}$ and actual target RFS $\mathcal{X}_{k}^{a}$, respectively, $\uplus$ denotes the disjoint union, $\breve{x} \in \breve{\mathcal{X}}_{k}, c \in \mathcal{X}_{k}^{f}$ and $x \in \mathcal{X}_{k}^{a}$. Since the measurements generated by false targets are independent of the clutter state $c, \nu_{k}^{(0)}(\cdot)$ is completely determined by the mean number of false targets $N_{k}^{(0)}$. Hence, the $\lambda$-PHD filter jointly propagates $\nu_{k}^{(1)}(\cdot)$ and $N_{k}^{(0)}$ over time. More details about the $\lambda$-PHD filter can be found in [36], [38].

In this work, at each sensor $i \in \mathcal{E}$ and every STI $k \in \mathcal{T}_{i}$, the $\lambda$-PHD filter is first updated using the received measurement $\mathcal{Z}_{i, k}$ based on the GM implementation. Then, the estimated clutter rate is computed by $\hat{r}_{i, c}=N_{i, k}^{(0)} p_{D, k}^{(0)}$, where $p_{D, k}^{(0)}$ stands for the detection probability of false targets. Finally, $\hat{r}_{i, c}$ is exploited in the CPHD filter to obtain the posterior cardinality distribution $\rho_{i, k}(n)$ and intensity function $\nu_{i, k}(x)$.

\section{B. Unknown Detection Probability Case}

In [37], the standard PHD filter was extended to deal with unknown detection probability by exploiting an augmented state model. The resulting augmented PHD filter recursively propagates the intensity function $\underline{\nu}_{k}(x, a)$ of the multitarget RFS $\underline{\mathcal{X}}_{k}$ containing information on the kinematic state $x$ and detection probability $a$. A closed-form solution to the augmented PHD recursion named the Beta GM PHD (BGMPHD) filter was derived in [36], where the Gaussian and Beta distributions are employed to model the kinematic state and detection probability, respectively.

In the present paper, the BGM-PHD filter is first performed at each sensor $i \in \mathcal{E}$ and every STI $k \in \mathcal{T}_{i}$. As a result, the intensity function $\underline{\nu}_{i, k}(x, a)$ is represented by $J_{i, k}$ BetaGaussian terms with positive weights $\alpha_{i, k}^{j}, j=1, \cdots, J_{i, k}$, i.e.,

$$
\underline{\nu}_{i, k}(x, a)=\sum_{j=1}^{J_{i, k}} \alpha_{i, k}^{j} \mathcal{N}\left(x ; \mu_{i, k}^{j}, \Sigma_{i, k}^{j}\right) \beta\left(a ; s_{i, k}^{j}, t_{i, k}^{j}\right),
$$

where $\beta(\cdot ; s, t)$ denotes the Beta distribution with shape parameters $s$ and $t$. The equivalent detection probability at sensor $i$ is then calculated by

$$
p_{i, k}^{d}=\frac{\sum_{j=1}^{J_{i, k}} \alpha_{i, k}^{j} \xi_{i, k}^{j}}{\sum_{j=1}^{J_{i, k}} \alpha_{i, k}^{j}},
$$

where $\xi_{i, k}^{j}=\frac{s_{i, k}^{j}}{s_{i, k}^{j}+t_{i, k}^{j}}$ denotes the mean of the $j$-th Beta term $\beta\left(a ; s_{i, k}^{j}, t_{i, k}^{j}\right)$. With the help of $p_{i, k}^{d}$, the posterior intensity function $\nu_{i, k}(x)$ and cardinality distribution $\rho_{i, k}(n)$ can be obtained by updating the local CPHD filter.

\section{Simulation Results}

This section provides two simulations examples to test the approaches proposed in Sections III and IV. In both examples, a sensor network consisting of 20 sensors S1-S20 with diameter $D_{m}=5$ is deployed in the surveillance region $[-2 \mathrm{~km}, 2 \mathrm{~km}] \times[-2 \mathrm{~km}, 2 \mathrm{~km}]$. The sampling rates of sensors S1-S10 and sensors S11-S20 are 2s and 3s, respectively. All sensors start sampling at the initial time $\tau=1 \mathrm{~s}$. At each example, 100 trials are carried out. The duration time of each trial is $120 \mathrm{~s}$. The network optimal subpattern assignment (NOSPA) and time-averaged N-OSPA (TN-OSPA) errors [48] are used throughout the examples to evaluate the tracking performance. The corresponding cut-off parameter and order parameter (see [51] for the meaning of these parameters) are set as $c=200 \mathrm{~m}$ and $p=2$, respectively.

\section{A. Linear Example With Known Model Parameters}

In this example, each target follows a constant velocity dynamic model and each sensor generates linear measurements. The target state is a vector $x_{k}=\left[p_{k}^{x}, p_{k}^{y}, v_{k}^{x}, v_{k}^{y}\right]^{T}$ of position $\left[p_{k}^{x}, p_{k}^{y}\right]^{T}$ and velocity $\left[v_{k}^{x}, v_{k}^{y}\right]^{T}$. The single target Markov density is specified by $\varphi_{k \mid k-1}\left(x_{k} \mid x_{k-1}\right)=\mathcal{N}\left(x_{k} ; F_{k} x_{k}, Q_{k}\right)$ with

$$
F_{k}=\left[\begin{array}{cc}
\mathrm{I}_{2 \times 2} & \Delta \mathrm{I}_{2 \times 2} \\
0_{2 \times 2} & \mathrm{I}_{2 \times 2}
\end{array}\right], Q_{k}=\sigma_{v}^{2}\left[\begin{array}{cc}
\frac{\Delta^{4}}{4} \mathrm{I}_{2 \times 2} & \frac{\Delta^{3}}{2} \mathrm{I}_{2 \times 2} \\
\frac{\Delta^{3}}{2} \mathrm{I}_{2 \times 2} & \Delta^{2} \mathrm{I}_{2 \times 2}
\end{array}\right] \text {, }
$$

where $\mathrm{I}_{\mathrm{n} \times \mathrm{m}}$ and $0_{n \times m}$ stand for the $n \times m$ identity and zero matrices, respectively, $\Delta=1 s$ and $\sigma_{v}=1 \mathrm{~m} / \mathrm{s}^{2}$. The birth intensity function $\gamma_{k}$ is given by $\gamma_{k}=\sum_{j=1}^{4} \alpha_{\gamma} \mathcal{N}\left(x ; \mu_{\gamma}^{i}, \Sigma_{\gamma}\right)$ where $\alpha_{\gamma}=0.03$, $\mu_{\gamma}^{1}=[0,0,500 \mathrm{~m}, 0]^{T}, \mu_{\gamma}^{2}=[400 \mathrm{~m}, 0,-600 \mathrm{~m}, 0]^{T}, \mu_{\gamma}^{3}=$ $[-800 \mathrm{~m}, 0,-200 \mathrm{~m}, 0]^{T}, \mu_{\gamma}^{4}=[-200 \mathrm{~m}, 0,800 \mathrm{~m}, 0]^{T}, \Sigma_{\gamma}=$ $\operatorname{diag}\left([10 \mathrm{~m}, 10 \mathrm{~m} / \mathrm{s}, 10 \mathrm{~m}, 10 \mathrm{~m} / \mathrm{s}]^{\mathrm{T}}\right)^{2}$. The survival probability is a constant 0.98 .

If target $x_{k}$ is detected by the sensor $i$ at time $k$, then a noisy position measurement $z_{i, k}$ is generated according to the likelihood function $\psi_{i, k}\left(z_{i, k} \mid x_{k}\right)=\mathcal{N}\left(z_{i, k} ; H_{i, k} x_{k}, R_{i, k}\right)$ with

$$
H_{i, k}=\left[\begin{array}{ll}
\mathrm{I}_{2 \times 2} & 0_{2 \times 2}
\end{array}\right], H_{i, k}=\sigma_{\varepsilon}^{2} \mathrm{I}_{2 \times 2},
$$

where $\sigma_{\varepsilon}=10 \mathrm{~m}$. The FOV of each sensor is the surveillance region. The clutter rate and detection probability of all sensors are known a prior and set as $\lambda_{i, c}=10$ and $p_{i, k}^{d}=0.95, i=$ $1, \cdots, 20$, respectively.

Filters resulting form the timely fusion approach including the AA-CPHD with feedback (in short AA-CPHD-F), AACPHD with non-feedback (in short AA-CPHD-NF), AA-CC with feedback (in short AA-CC-F) and AA-CC with nonfeedback (in short AA-CC-NF) are compared with the noncooperative filter without communication and fusion operations. Each local CPHD filter truncates the cardinality distribution at $N_{\max }=50$, makes use of at most $100 \mathrm{GCs}$ to represent the intensity function, selects T-GCs using a weight threshold $r_{\text {gate }}=0.001$ and merges T-GCs with Mahalanobis distance below $4 \mathrm{~m}$. 


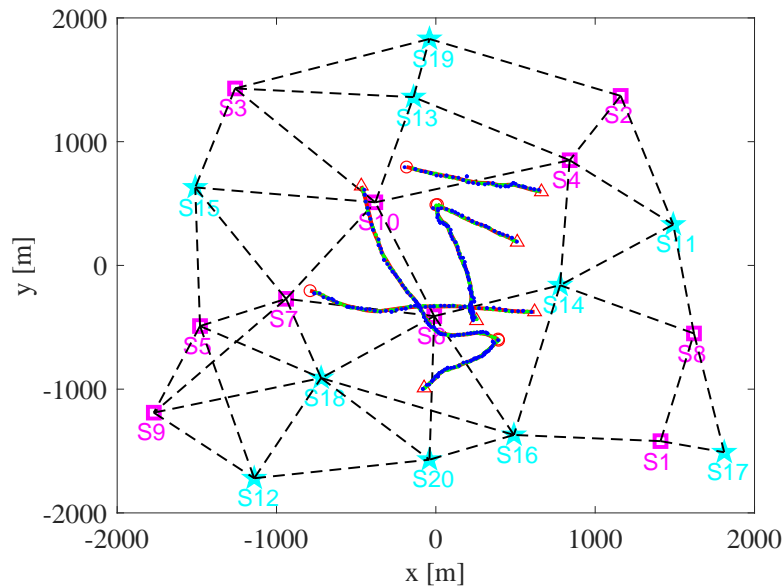

Fig. 4. Linear simulation scenario. Sensors are positioned at magenta squares and cyan pentagrams, respectively. Target trajectories are plotted by red lines with the start and end positions given by circles and triangles, respectively. Communication links are indicated by black dashed lines. The estimates of the non-cooperative and AA-CPHD-F filters are marked by blue and green points, respectively.

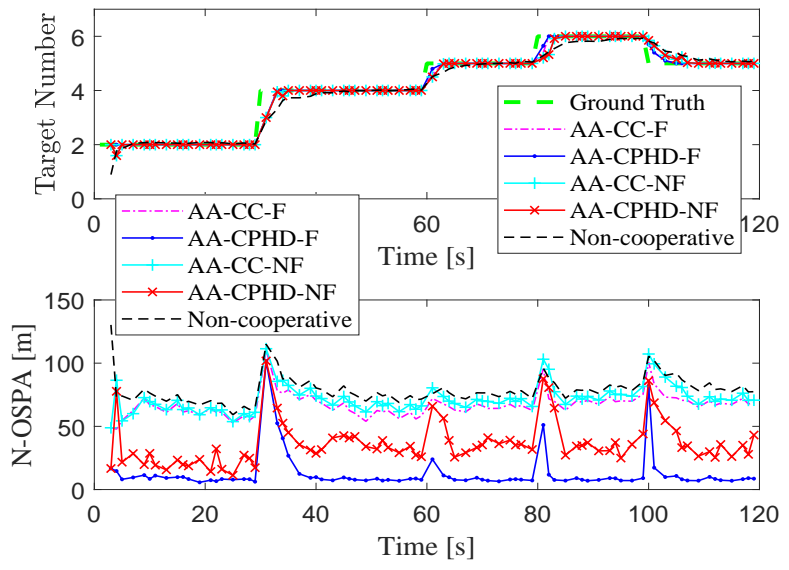

Fig. 5. Averaged target number estimates and N-OSPA errors versus time.

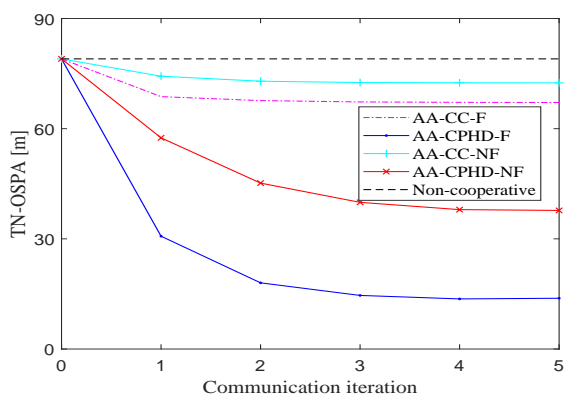

Fig. 6. Averaged TN-OSPA errors versus communication iteration.

The true trajectories and the sensor topology together with position estimates of the AA-CPHD-F filter (when $L=5$ ) and non-cooperative filter are plotted in Fig. 4. Fig. 5 presents the averaged target number estimates and N-OSPA errors for the filters under study. The results indicate the following findings:

- The proposed filters perform better than the noncooperative filter thanks to the AA fusion.

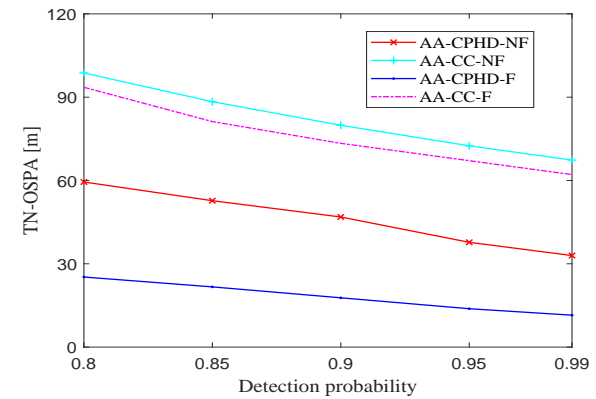

Fig. 7. Averaged TN-OSPA errors versus varying detection probability.

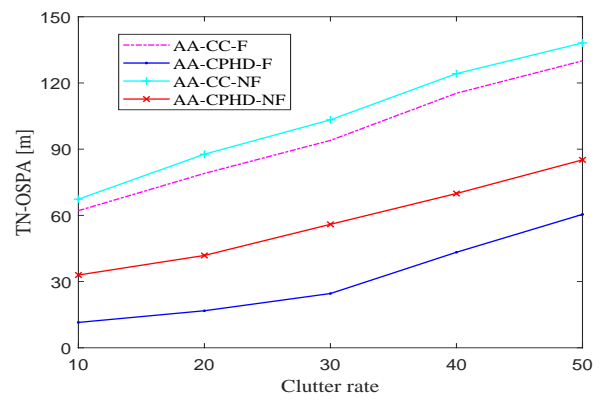

Fig. 8. Averaged TN-OSPA errors versus varying clutter rate.

- The feedback mode is superior to its non-feedback counterpart since the fusion outcome is helpful to improve the local estimates.

- The AA-CPHD fusion prefers over the AA-CC fusion, which can be attributed to the fact that more target-related information is used in the fusion operation.

Fig. 6 shows the TN-OSPA errors versus communication iteration for different filters. As expected, the TN-OSPA errors for different filters decrease with the increase of the number of communication iterations. We also test the TN-OSPA errors versus varying clutter rate and varying detection probability for the AA-CPHD-F, AA-CPHD-NF, AA-CC-F and AA-CCNF filters, respectively. The corresponding results are given in Figs. 7 and 8, which further consolidate the results in Fig. 5.

\section{B. Nonlinear Example With Unknown Model Parameters}

In this example, the single target state is given by $x_{k}=$ $\left[\tilde{x}_{k}^{T}, \omega_{k}\right]^{T}$ with position and velocity $\tilde{x}_{k}^{T}=\left[p_{k}^{x}, v_{k}^{x}, p_{k}^{y}, v_{k}^{y}\right]^{T}$ together with turn rate $\omega_{k}$. The state Markov density is specified by $\varphi_{k \mid k-1}\left(x_{k} \mid x_{k-1}\right)=\mathcal{N}\left(x_{k} ; F\left(\omega_{k}\right) x_{k}, Q_{k}\right)$, where

$$
F(\omega)=\left[\begin{array}{ccccc}
1 & \frac{\sin \omega \Delta}{\omega} & 0 & -\frac{1-\cos \omega \Delta}{\omega} & 0 \\
0 & \cos \omega \Delta & 0 & -\sin \omega \Delta & 0 \\
0 & \frac{1-\cos \omega \Delta}{\omega} & 1 & \frac{\sin \omega \Delta}{\omega} & 0 \\
0 & \sin \omega \Delta & 0 & \cos \omega \Delta & 0 \\
0 & 0 & 0 & 0 & 1
\end{array}\right],
$$

and $Q_{k}=\operatorname{diag}\left(\left[\mathrm{I}_{2} \otimes \mathrm{G}\right], \sigma_{u}^{2}\right)$ with

$$
\mathrm{G}=\left[\begin{array}{cc}
\frac{\sigma_{\omega}^{2}}{4} & \frac{\sigma_{\omega}^{2}}{2} \\
\frac{\sigma_{\omega}^{2}}{2} & \sigma_{\omega}^{2}
\end{array}\right],
$$






Fig. 9. Nonlinear simulation scenario. The estimates of the R-AA-CPHD-F filter for unknown clutter and detection parameters are plotted by green and cyan points, respectively. The other landmarks are consistent with Fig. 4.

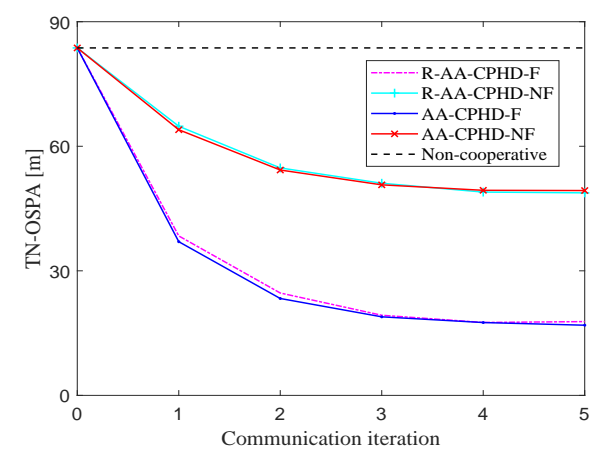

Fig. 10. Averaged TN-OSPA errors versus communication iterations for the unknown clutter rate case.

where $\otimes$ denotes the Kronecker product, $\Delta=1 \mathrm{~s}, \sigma_{\omega}=$ $1 \mathrm{~m} / \mathrm{s}^{2}$ and $\sigma_{u}=(\pi / 360) \mathrm{rad} / \mathrm{s}$. The birth intensity function $\gamma_{k}$ is given by $\gamma_{k}=\sum_{j=1}^{4} \alpha_{\gamma}^{i} \mathcal{N}\left(x ; \mu_{\gamma}^{i}, \Sigma_{\gamma}\right)$ where $\alpha_{\gamma}^{1}=\alpha_{\gamma}^{2}=0.02, \alpha_{\gamma}^{3}=\alpha_{\gamma}^{4}=0.03, \mu_{\gamma}^{1}=$ $[900 \mathrm{~m}, 0,400 \mathrm{~m}, 0,0]^{T}, \mu_{\gamma}^{2}=[-300 \mathrm{~m}, 0,700 \mathrm{~m}, 0,0]^{T}, \mu_{\gamma}^{3}=$ $[-400 \mathrm{~m}, 0,0,0,0]^{T}, \mu_{\gamma}^{4}=[250 \mathrm{~m}, 0,750 \mathrm{~m}, 0,0]^{T}, \Sigma_{\gamma}=$ $\operatorname{diag}\left([20 \mathrm{~m}, 20 \mathrm{~m} / \mathrm{s}, 20 \mathrm{~m}, 20 \mathrm{~m} / \mathrm{s}, 4(\pi / 180) \mathrm{rad} / \mathrm{s}]^{\mathrm{T}}\right)^{2}$. If detected by the sensor $i$ at time $k$, the single target measurement model is determined by

$$
z_{i, k}=\left[\frac{\arctan \left(\frac{p_{k}^{x}-p_{i}^{x}}{p_{k}^{y}-p_{i}^{y}}\right)}{\sqrt{\left(p_{k}^{x}-p_{i}^{x}\right)^{2}+\left(p_{k}^{y}-p_{i}^{y}\right)^{2}}}\right]+\varepsilon_{i, k}
$$

where $\left[p_{i}^{x}, p_{i}^{y}\right]^{T}$ denotes the location of sensor $i, \varepsilon_{i, k} \sim$ $\mathcal{N}\left(\cdot ; 0_{2 \times 1}, R_{i, k}\right), R_{i, k}=\operatorname{diag}\left(\left[\sigma_{\theta}^{2}, \sigma_{r}^{2}\right]^{T}\right), \sigma_{\theta}=(\pi / 180) \mathrm{rad} / \mathrm{s}$ and $\sigma_{r}=10 \mathrm{~m}$. The FoV of each sensor is a circle with the sensor's location as the center and $5000 \mathrm{~m}$ as the radius, which can cover the surveillance region.

We demonstrate the performance of the robust bootstrap approach in two separate cases. In particular, the AA-CPHD$\mathrm{F}$ and AA-CPHD-NF filters are considered to accommodate unknown model parameters on account of their preferable performance, yielding the robust AA-CPHD-F (in short R-AA-

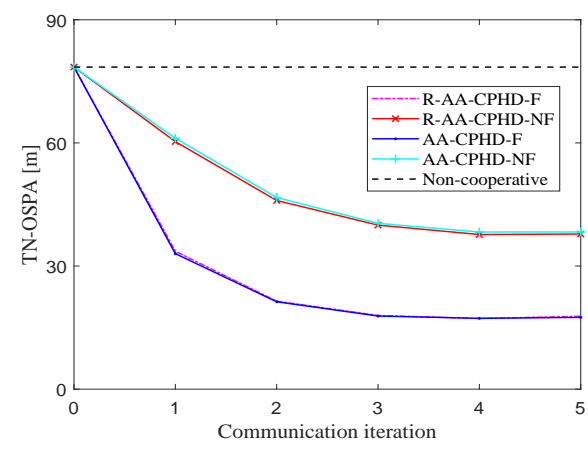

Fig. 11. Average TN-OSPA errors versus communication iterations for the unknown detection probability case.

CPHD-F) filter and the robust AA-CPHD-NF (in short R-AACPHD-NF) filter. Each case also contains a comparison with the AA-CPHD-F, AA-CPHD-NF and non-cooperative filters.

For the case of unknown clutter rate, the mean clutter rate for each sensor is 20. For the case of unknown detection probability, the detection probability for each sensor is a constant 0.9. These information is not known to the R-AACPHD-F and R-AA-CPHD-NF filters. The other parameters used in the local PHD estimators follow the choice of [36]. The local CPHD filters adopt the unscented transform to handle the system nonlinearity.

Fig. 9. plots the position estimates of the R-AA-CPHD-F (when $L=5$ ) filter and non-cooperative filters for two cases in addition to the true trajectories as well as the sensor topology. The TN-OSPA errors versus communication iteration for the two cases are shown in Figs. 10 and 11. As shown, the R-AACPHD-F filter performs almost as well as its benchmark filter, i.e., the AA-CPHD-F filter and so does the R-AA-CPHD-NF filter. These results suggest that the proposed robust bootstrap approach can deal with unknown clutter and detection parameters without difficulty.

\section{CONCLUSION}

In this paper, two DMT approaches are proposed to handle different sampling rates among sensors. In the first timely fusion approach, the CPHD filter is carried out at each sensor for generating local estimates, while the AA fusion is employed to combine local estimates from different sensors. The information exchange among sensors follows a partial flooding protocol, in which either intensity functions or cardinality distributions with respect to local posteriors are broadcast throughout the network. Besides the feedback fusion-filtering mode, where the fusion outcome is utilized to update local estimates, a non-feedback alternative is also investigated for the purpose of avoiding/reducing sensor data delay. The second robust bootstrap approach is an extension of the timely fusion approach, which takes unknown clutter and detection parameters into consideration. The centerpiece of the robust bootstrap approach is the local bootstrap filter consisting of a PHD estimator and a CPHD filter. Specifically, the PHD estimator is run first and its estimates of the model parameters are then provided to the CPHD filter to generate the local posterior estimates. Numerical simulation are performed and 
their results demonstrate the validity of the proposed timely fusion and robust bootstrap approaches. Our future work will consider extensions of the proposed approaches to labeled RFS filters [52], [53] and resource-limited sensor networks [54], [55].

\section{REFERENCES}

[1] I. F. Akyildiz, W. Su, Y. Sankarasubramaniam, and E. Cayirci, "A survey on sensor networks," IEEE Commun. Mag., vol. 40, no. 8, pp. 102-114, Aug. 2002.

[2] C.-Y. Chong and S. P. Kumar, "Sensor networks: evolution, opportunities, and challenges," Proc. IEEE, vol. 91, no. 8, pp. 1247-1256, Aug. 2003.

[3] M. Üney, D. E. Clark, and S. J. Julier, "Distributed fusion of PHD filters via exponential mixture densities," IEEE J. Sel. Topics Signal Process., vol. 7, no. 3, pp. 521-531, Jun. 2013.

[4] G. Battistelli, L. Chisci, C. Fantacci, A. Farina, and A. Graziano, "Consensus CPHD filter for distributed multitarget tracking," IEEE J. Sel. Topics Signal Process., vol. 7, no. 3, pp. 508-520, Jun. 2013.

[5] T. Li, F. Hlawatsch, and P. M. Djurić, "Cardinality-consensus-based PHD filtering for distributed multitarget tracking," IEEE Signal Process. Lett., vol. 26, no. 1, pp. 49-53, Jan. 2019

[6] L. Gao, G. Battistelli, and L. Chisci, "Event-triggered distributed multitarget tracking," IEEE Trans. Signal Inf. Process. Netw., vol. 5, no. 3, pp. 570-584, Jul. 2019.

[7] R. P. Mahler, Statistical multisource-multitarget information fusion Norwell, MA, USA: Artech House, 2007.

[8] R. P. Mahler, Advances in statistical multisource-multitarget information fusion. Norwell, MA, USA: Artech House, 2014

[9] R. P. Mahler, "Multitarget Bayes filtering via first-order multitarget moments," IEEE Trans. Aerosp. Electron. Syst., vol. 39, no. 4, pp. $1152-$ 1178, Oct. 2003.

[10] R. Mahler, "PHD filters of higher order in target number," IEEE Trans. Aerosp. Electron. Syst., vol. 43, no. 4, pp. 1523-1543, Oct. 2007.

[11] B.-N. Vo and W.-K. Ma, "The Gaussian mixture probability hypothesis density filter," IEEE Trans. Signal Process., vol. 54, no. 11, pp. 40914104, Nov. 2006.

[12] B.-T. Vo, B.-N. Vo, and A. Cantoni, "Analytic implementations of the cardinalized probability hypothesis density filter," IEEE Trans. Signal Process., vol. 55, no. 7, pp. 3553-3567, Jul. 2007.

[13] B.-N. Vo, S. Singh, and A. Doucet, "Sequential Monte Carlo methods for multitarget filtering with random finite sets," IEEE Trans. Aerosp. Electron. Syst., vol. 41, no. 4, pp. 1224-1245, Oct. 2005.

[14] T. Li, J. M. Corchado, and S. Sun, "On generalized covariance intersection for distributed phd filtering and a simple but better alternative," in Proc. Int. Conf. Inf. Fusion., Xi'an, China, Jul. 2017, pp. 1-8.

[15] A. K. Gostar, R. Hoseinnezhad, and A. Bab-Hadiashar, "CauchySchwarz divergence-based distributed fusion with poisson random finite sets," in Proc. Int. Conf. Comput. Appl. Inf. Secur., Chiang Mai, Thailand, Oct. 2017, pp. 112-116.

[16] L. Gao, G. Battistelli, and L. Chisci, "Multiobject fusion with minimum information loss," IEEE Signal Process. Lett., vol. 27, pp. 201-205, Jan. 2020.

[17] D. Clark, S. Julier, R. Mahler, and B. Ristic, "Robust multi-object sensor fusion with unknown correlations," in Proc. IEEE Sens. Signal Process. Defence., London, U.K., 2010, pp. 1-5.

[18] M. Üney, J. Houssineau, E. Delande, S. J. Julier, and D. E. Clark, "Fusion of finite-set distributions: Pointwise consistency and global cardinality," IEEE Trans. Aerosp. Electron. Syst., vol. 55, no. 6, pp. 2759-2773, Dec. 2019.

[19] T. Li, H. Fan, J. García, and J. M. Corchado, "Second-order statistics analysis and comparison between arithmetic and geometric average fusion: Application to multi-sensor target tracking," Inf. Fusion., vol. 51, pp. 233-243, Nov. 2019.

[20] T. Li, "Why mixture?" TechRxiv.15082113, 2021.

[21] K. Da, T. Li, Y. Zhu, and Q. Fu, "Gaussian mixture particle jumpMarkov-CPHD fusion for multitarget tracking using sensors with limited views," IEEE Trans. Signal Inf. Process. Netw., vol. 6, pp. 605-616, Aug. 2020.

[22] W. Yi, G. Li, and G. Battistelli, "Distributed multi-sensor fusion of PHD filters with different sensor fields of view," IEEE Trans. Signal Process., vol. 68 , pp. 5204-5218, Sep. 2020.
[23] T. Li, V. Elvira, H. Fan, and J. M. Corchado, "Local-diffusion-based distributed SMC-PHD filtering using sensors with limited sensing range," IEEE Sensors J., vol. 19, no. 4, pp. 1580-1589, Feb. 2018.

[24] L. Gao, G. Battistelli, L. Chisci, and A. Farina, "Fusion-based multidetection multi-target tracking with random finite sets," IEEE Trans. Aerosp. Electron. Syst., 2021, In press.

[25] T. Li and F. Hlawatsch, "A distributed particle-PHD filter using arithmetic-average fusion of Gaussian mixture parameters," Inf. Fusion., vol. 73, pp. 111-124, Sep. 2021.

[26] T. Li, M. Mallick, and Q. Pan, "A parallel filtering-communication-based cardinality consensus approach for real-time distributed phd filtering," IEEE Sens. J., vol. 20, no. 22, pp. 13 824-13 832, Nov. 2020

[27] T. Li, J. M. Corchado, and S. Sun, "Partial consensus and conservative fusion of Gaussian mixtures for distributed PHD fusion," IEEE Trans. Aerosp. Electron. Syst., vol. 55, no. 5, pp. 2150-2163, Oct. 2018.

[28] H. Kim, K. Granström, L. Gao, G. Battistelli, S. Kim, and H. Wymeersch, "5g mmwave cooperative positioning and mapping using multimodel PHD filter and map fusion," IEEE Trans. Wireless Commun., vol. 19 , no. 6, pp. 3782-3795, Jun. 2020.

[29] R. K. Ramachandran, N. Fronda, and G. Sukhatme, "Resilience in multirobot multi-target tracking with unknown number of targets through reconfiguration," IEEE Trans. Control. Netw. Syst., 2021, In press.

[30] Q. Yang, W. Yi, and M. Mallick, "A decentralized PHD filter for multitarget tracking in asynchronous multi-static radar system," in Proc. IEEE Radar Conf., Florence, Italy, Sep. 2020, pp. 1-6.

[31] G. Li, W. Yi, and L. Kong, "A real-time fusion algorithm for asynchronous sensor system with PHD filter," in Proc. IEEE Intl. Conf. Control Autom. Inf. Sci., Chiang Mai, Thailand, Oct. 2017, pp. 134139

[32] G. Li, W. Yi, M. Jiang, and L. Kong, "Distributed fusion with PHD filter for multi-target tracking in asynchronous radar system," in Proc. IEEE Radar Conf., Seattle, WA, USA, May. 2017, pp. 1434-1439.

[33] S. Liu, H. Shen-tu, H. Chen, D. Peng, and Y. Shi, "Asynchronous multisensor fusion multi-target tracking method," in Proc. IEEE Intl. Conf. Control Autom., Anchorage, Alaska, USA, Jul. 2018, pp. 459-463.

[34] G. Li, W. Yi, S. Li, B. Wang, and L. Kong, "Asynchronous multi-rate multi-sensor fusion based on random finite set," Signal Process., vol 160, pp. 113-126, Jul. 2019

[35] G. Li, G. Battistelli, L. Chisci, and L. Kong, "Distributed multi-target tracking over an asynchronous multi-sensor network," in Proc. IEEE Radar Conf., Florence, Italy, Sep. 2020, pp. 1-6.

[36] R. P. Mahler, B.-T. Vo, and B.-N. Vo, "CPHD filtering with unknown clutter rate and detection profile," IEEE Trans. Signal Process., vol. 59, no. 8, pp. 3497-3513, Aug. 2011.

[37] R. Mahler and A. El-Fallah, "CPHD filtering with unknown probability of detection," in Signal Process., Sens. Fusion, Target Recognit. XIX, SPIE Proc., vol. 7697, Apr. 2010.

[38] R. Mahler and A. El-Fallah, "CPHD and PHD filters for unknown backgrounds, part iii: tractable multitarget filtering in dynamic clutter,' in Signal Data Process. Small Targets, SPIE Proc., vol. 7698, Apr.

[39] S. H. Rezatofighi, S. Gould, B. T. Vo, B.-N. Vo, K. Mele, and R. Hartley, "Multi-target tracking with time-varying clutter rate and detection profile: Application to time-lapse cell microscopy sequences," IEEE Trans. Med. Imag., vol. 34, no. 6, pp. 1336-1348, Jun. 2015.

[40] M. Beard, B.-T. Vo, and B.-N. Vo, "Multitarget filtering with unknown clutter density using a bootstrap GMCPHD filter," IEEE Signal Process. Lett., vol. 20, no. 4, pp. 323-326, Apr. 2013.

[41] R. Mahler and B.-T. Vo, "An improved CPHD filter for unknown clutter backgrounds," in Signal Process., Sens. Fusion, Target Recognit. XXIII SPIE, Proc., vol. 9091, Jun. 2014.

[42] C. Li, W. Wang, T. Kirubarajan, J. Sun, and P. Lei, "PHD and CPHD filtering with unknown detection probability," IEEE Trans. Signal Process., vol. 66, no. 14, pp. 3784-3798, Jul. 2018.

[43] X. Chen, R. Tharmarasa, M. Pelletier, and T. Kirubarajan, "Integrated clutter estimation and target tracking using Poisson point processes," IEEE Trans. Aerosp. Electron. Syst., vol. 48, no. 2, pp. 1210-1235, Apr. 2012.

[44] F. Lian, C. Han, and W. Liu, "Estimating unknown clutter intensity for PHD filter," IEEE Trans. Aerosp. Electron. Syst., vol. 46, no. 4, pp. 2066-2078, Dec. 2010.

[45] I. Schlangen, V. Bharti, E. Delande, and D. E. Clark, "Joint multi-object and clutter rate estimation with the single-cluster PHD filter," in Proc. IEEE. Symp. Biomed. Imag., Melbourne, Australia, Apr. 2017, pp. 10871091.

[46] K. Da, T. Li, Y. Zhu, H. Fan, and Q. Fu, "Kullback-Leibler averaging for multitarget density fusion," in Proc. Int. Symp. Distrib. Comput. Artif. Intell., Avila, Spain, Jun. 2019, pp. 253-261. 
[47] K. Da, T. Li, Y. Zhu, H. Fan, and Q. Fu, "Recent advances in multisensor multitarget tracking using random finite set," Frontiers Inf. Technol. Electron. Eng., vol. 22, no. 1, pp. 5-24, Jan. 2021.

[48] T. Li, X. Wang, Y. Liang, and Q. Pan, "On arithmetic average fusion and its application for distributed multi-Bernoulli multitarget tracking," IEEE Trans. Signal Process., vol. 68, pp. 2883-2896, Apr. 2020.

[49] T. Li, J. M. Corchado, and J. Prieto, "Convergence of distributed flooding and its application for distributed bayesian filtering," IEEE Trans. Signal Inf. Process. Netw., vol. 3, no. 3, pp. 580-591, Sep. 2016.

[50] H. Van Nguyen, H. Rezatofighi, B.-N. Vo, and D. C. Ranasinghe, "Distributed multi-object tracking under limited field of view sensors," IEEE Trans. Signal Process., 2021, In press.

[51] D. Schuhmacher, B.-T. Vo, and B.-N. Vo, "A consistent metric for performance evaluation of multi-object filters," IEEE Trans. Signal Process., vol. 56, no. 8, pp. 3447-3457, Aug. 2008.

[52] B.-N. Vo, B.-T. Vo, and D. Phung, "Labeled random finite sets and the bayes multi-target tracking filter," IEEE Trans. Signal Process., vol. 62, no. 24, pp. 6554-6567, Dec. 2014

[53] T. Kropfreiter, F. Meyer, and F. Hlawatsch, "A fast labeled multiBernoulli filter using belief propagation," IEEE Trans. Aerosp. Electron. Syst., vol. 56, no. 3, pp. 2478-2488, Jun 2020.

[54] M. Mallick, V. Krishnamurthy, and B.-N. Vo, Sensor Management for Large-Scale Multisensor-Multitarget Tracking. Hoboken, NJ, USA: Wiley, 2014

[55] J. Yan, W. Pu, S. Zhou, H. Liu, and M. S. Greco, "Optimal resource allocation for asynchronous multiple targets tracking in heterogeneous radar networks," IEEE Trans. Signal Process., vol. 68, pp. 4055-4068, Jul. 2020. 